\title{
A FEW PROVISIONAL CONCLUSIONS FROM OBSERVATIONS ON ALIGNMENT OF COBALT NUCLEI
}

\author{
by C. J. GORTER, H. A. TOLHOEK *, O. J. POPPEMA **, \\ M. J. STEENLAND and J. A. BEUN
}

Suppl. $N^{\circ} 104 a$ of the Communications of the Kamerlingh Onnes Laboratory, Leiden, Nederland

\section{Synopsis}

From the observed anisotropy of the gamma-ray emission of aligned ${ }^{60} \mathrm{Co}$ the magnetic moment of this nucleus is estimated to be 3.2 nuclear magnetons. From the dependence of the anisotropy on a magnetic field it is concluded that the hyperfine splitting of ${ }^{59} \mathrm{Co}$ in the salt concerned is $0.13 \mathrm{~cm}^{-1}$ between consecutive levels.

It is possible to derive a few interesting conclusions from recent rather crude observations ${ }^{1}$ ) on alignment of ${ }^{60} \mathrm{Co}$ nuclei in diluted hydrated cobalt ammonium sulphate, $\mathrm{Co}_{0.05} \mathrm{Zn}_{0.95}\left(\mathrm{NH}_{4}\right)_{2}\left(\mathrm{SO}_{4}\right)_{2} \cdot 6 \mathrm{H}_{2} \mathrm{O}$. When the salt was demagnetized from a very strong field in the $\mathrm{K}_{I}$-direction an anisotropy of about $12 \%$ was found in the gamma-ray emission of ${ }^{60} \mathrm{Co}$. In agreement with $\mathrm{D}$ e u t s ch 's interpretation ${ }^{2}$ ) of the emission as due to quadrupole processes, the intensity had a minimum in the $\mathrm{K}_{I}$-direction which presumably makes an angle of about $34^{\circ}$ with the axes of the crystalline fields acting on the Co-ions.

From the magnitude of the anisotropy one may estimate the magnetic moment of the ${ }^{60} \mathrm{Co}$-nucleus in the following way. The electronic moment $J=\frac{1}{2}$ is completely orientated in the original magnetic field giving an entropy $k \ln 8$, corresponding to eight equivalent orientations of the ${ }^{59} \mathrm{Co}$-nucleus. In agreement with B leaney's analysis ${ }^{3}$ ) we suppose to find eight equidistant doubly degenerate levels after demagnetizing. If the entropy is un-altered the ratio of the occupation of consecutive levels is exp (0.67). If we assume ${ }^{2}$ ) the ${ }^{60} \mathrm{Co}$-nucleus to have $I=5$ and to give one beta process determined by a nuclear matrix element of vectorial character $(5 \rightarrow 4)$ and two quadrupolar gamma processes

*) Instituut voor Theoretische Natuurkunde der Rijksuniversiteit, Utrecht.

**) Natuurkundig Laboratorium der Rijksuniversiteit, Groningen. 
$(4 \rightarrow 2 \rightarrow 0)$, we may explain the observed magnitude of the anisotropy of the gamma-rays on the basis of a Boltzmann factor $\exp (0.32)$ over the consecutive ${ }^{60} \mathrm{Co}$-levels. This indicates that the ratio between the $g$-values of ${ }^{60} \mathrm{Co}$ and ${ }^{59} \mathrm{Co}$ is $32 / 67$. The $g$-factor of ${ }^{59} \mathrm{Co}$ being known, this gives $\mu=g I=3.2$ nuclear magnetons. The uncertainty may be of the order of 0.3 magnetons. Prof. F. E. $\mathrm{S}$ i m o n kindly informs us that the independent Oxford investigations ${ }^{4}$ ) with a crystal containing also copper ions lead to $\mu=3.0 \pm 0.5$. Prof. C. J. B a k k e r of the University of Amsterdam kindly informs us that Mr. P. F. A. K lin ke n berg has calculated the magnetic moment of ${ }^{60} \mathrm{Co}$ on the basis of the shell model. Any coupling between an $f_{7 / 2}$-proton and a $p_{3 / 2}$-neutron gives $\mu=3.88$, if the $g$-value of free protons and neutrons are used, and $\mu=3.51$, if the $g$ 's are adjusted for the case of bound particles. Our value lies between the Oxford result and the theoretical predictions.

It was found experimentally that the effect decreases to half its value in a constant field of 250 oersteds in the $\mathrm{K}_{I}$-direction. In such a field a considerable resulting nuclear polarization must exist. In view of the symmetry character of the gamma-rays this will not be directly observable and the anisotropy will only be effected in so far as the temperature $T$ has increased. A small anisotropy will be approximately proportional to $T^{-2}$. Since the increase of temperature is determined by the ratio of Zeeman effect and hyperfine splitting of ${ }^{59} \mathrm{Co}$, we may estimate the hyperfine splitting. This gives a separation of $0.13 \pm 0.01 \mathrm{~cm}^{-1}$ between consecutive levels of ${ }^{59} \mathrm{Co}$ in good agreement with the value 0.12 $\mathrm{cm}^{-1}$ deduced by $\mathrm{Ble}$ a n e $\mathrm{y}^{3}$ ) from the hyperfine structure of paramagnetic resonance in this salt.

Accurate observations of the angular distribution of the radiation and of correlations are being prepared.

Received 23-1-52.

\section{REFERENCES}

1) Gorter, C. J. Versl. Kon. Ned. Akad. Wet, 15 (1951) 104. G or ter, C. J. Pop pem a, O. J., S t e e $\mathrm{l}$ a $\mathrm{nd}$, M. J. and Be un, J. A., Commun. Kamerlingh Onnes Lab., Leiden, $N^{\circ} 287 b$, Physica, Amsterdam, 17 (1951) 1050.

2) De ut s ch, M. and S c h a rff-Gold ha ber, G. Phys. Rev., (2) 83 (1951) 1059.

3) Bl e a n e y, B., Proc. phys. Soc., London A 64 (1951) 316; Phil. Mag. (7) 42 (1951) 441.

4) Daniels, J. M., Grace, M. A. and R obins o n, F. N. H., Nature, London 168 (1951) 780; B l e aney, B., Daniels, J. M. Grace, M. A. Halban, H., Kurti, N. and Robinson, F. N. H., in the press. 